\title{
RADIATIVE SIGNATURES OF NEUTRON BEAMS IN AGN
}

\author{
IFEANYI E. EKEJIUBA \\ Department of Physics \& Astronomy, Georgia State University, Atlanta, Georgia 30303, USA \\ and Department of Physics, Federal University of Technology, Yola, Nigeria
}

\begin{abstract}
The escape of relativistic neutrons from their production region can have various consequencies for the morphology of active galaxies. The phenomena of luminosity gaps and radio jet lighting in extragalactic radio sources (EGRSs) fit into the model that employs relativistic neutrons as the vector for particle and energy transport out of the central engines of AGNs. The central radio gaps reveal themselves as regions of relativistic neutron beam transport. The relativistic neutrons, which decay in flight after traveling for $\sim 10^{3} \gamma_{n} \mathrm{~s}$, produce secondaries which are responsible for the radio jet lighting and the associated phenomena in EGRSs.
\end{abstract}

\section{The Radiative Signatures of Relativistic Neutrons}

The accretion disc region favors the production of relativistic neutrons with the following reactions as the dominant production mechanisms (Ekejiuba \& Okeke 1993a): ${ }^{4} \mathrm{He}+\gamma \rightarrow 2 p+2 n ; p+p \rightarrow p+n+\pi^{+} \rightarrow p+n+e^{+}+\nu ; p+\gamma \rightarrow$ $n+\pi^{+} \rightarrow n+e^{+}+\nu$ where $\mathbf{p}, \mathbf{n}, \pi$, e and $\nu$ refer to proton, neutron, pion, electron and neutrino, respectively. The relativistic neutrons produced escape beyond the accretion zone and travel ballistically after an initial collimation of the precursor proton beam and decay after traveling for $\sim 10^{13} \gamma_{n}$ s to produce protons, electrons and neutrinos: $n \rightarrow p+e^{-}+\bar{\nu}$.

The interactions of the secondary particles with entrained ambient matter and magnetic field produce the observed radiations in AGNs. Three channels of interaction dominate: (a) synchrotron emission of the decay-produced electrons, with $\gamma_{e} \sim 2 k \gamma_{n}$, where $k$ is the neutron- to electron-energy conversion efficiency (e.g., Ekejiuba \& Okeke 1993b); (b) interaction of the decay-produced relativistic protons with entrained ambient (cold!) protons to liberate relativistic electrons, which spiral in the background magnetic field producing synchrotron emissions, with $\gamma_{e} \sim 60 \gamma_{p}$ where $\gamma_{p}$ is the energy of the leading proton; and (c) elastic Coulomb interactions between relativistic protons and non-relativistic or mildly relativistic electrons during which the electrons are boosted to the relativistic range adequate for synchrotron radiation, with $\gamma_{e} \sim 10 \gamma_{p}^{1 / 3}$ (e.g., Ekejiuba \& Okeke 1993b). Due to their neutrality, the relativistic neutrons create a luminosity gap (the so-called central radio gap) in the region where the collimated neutron beam is traveling before the neutrons decay.

\section{References}

Ekejiuba, I.E. \& Okeke, P.N. 1993a, ApJ, 413, 110.

Ekejiuba, I.E. \& Okeke, P.N. 1993b, ApJ, in press

T. J.-L. Courvoisier and A. Blecha: Multi-Wavelength Continuum Emission of AGN, 345.

(C) 1994 IAU. Printed in the Netherlands. 